\section{La variabile tempo nella trasformazione: emergenza e fragilità}

Luigi Spinelli

Politecnico di Milano, Dipartimento di Architettura e Studi Urbani (luigimario.spinelli@polimi.it)

«[... 2) L'industriale moderno deve tendere ad isolare nello spazio la fabbrica e a fonderla col paesaggio, in modo da emanciparsi dall'urbanistica cittadina. Questa è la condizione principale affinché la fabbrica assuma e mantenga un suo definito valore artistico ed una missione sociale. 3) Non realizzare mai programmi parziali se non si possiede un programma generale del quale si è convinti ed al quale si vuol tener fede nel più breve tempo possibile. 4) Ricordare che le costruzioni sporadiche o provvisorie e le migliorie parziali sono indice di incertezza e di debolezza. Queste esitazioni edilizie creano confusioni nella produzione e si risolvono in danni economici. 5) Meglio disfarsi a tempo e con coraggio di una vecchia costruzione irrazionale e superata, piuttosto che sacrificare l'estetica futura ad una pericolosa avarizia iniziale [...]». Questi alcuni dei principi fissati da Giuseppe Pagano nel «Decalogo agli industriali» sul numero 175 di Casabella-Costruzioni nel 1942.

Era difficile immaginare una fase ulteriore, quasi un secolo dopo, di superamento della città industriale e di re-immissione di queste presenze nel territorio urbanizzato. A metà degli anni '70 appariranno nuove attenzioni al riuso del patrimonio industriale esistente, per le quali, a fronte di interessi immobiliari e di fenomeni di discontinuità della forma urbana, le trasformazioni verranno gestite attraverso procedure urbanistiche meno attente al manufatto architettonico. Con la nascita in Italia nel 1985 dell'Istituto di Cultura Materiale e Archeologia Industriale (ICMAI), si avvierà un'azione di sensibilizzazione verso questo patrimonio, fino alla costituzione nel 1994 da parte del Ministero di una Commissione Nazionale per i Beni Culturali Industriali: vengono considerati patrimonio industriale i complessi o edifici rappresentativi di una emblematica industrializzazione legata ad un'area geografica o culturale. Il termine 'Archeologia', paradossalmente accostato all'aggettivo 'Industriale', legato ad un'idea di modernità, solleva l'urgenza di una definizione di campo e richiede continue revisioni delle soglie cronologiche. Negli anni '80 l'attenzione si rivolge in modo deciso al recupero del senso della storia, al culto della 'memoria', con l'inizio di un'attività di censimento e rilievo, catalogazione e recupero di un patrimonio al quale verrà applicata l'etichetta di 'bene culturale'. Un concetto, questo, vario e non bene definito nei suoi caratteri di individuazione e gestione, sul quale si applicheranno con un'incessante operatività ricercatori, storici, restauratori, critici, con la produzione di indagini scientifiche, riscoperte storiografiche, saggi critici, convegni e seminari, alla convergente ricerca di mettere ordine tra i molteplici aspetti della sua definizione e di un approccio sistematico ai tempi e ai modi del recupero. Le categorie del 'restauro', della 'conservazione', del 'riuso', della 'riqualificazione' si confonderanno, alternandosi al capezzale di quelle architetture che in qualche modo vengono individuate come degne di essere curate e restituite nella maggiore integrità possibile alle future generazioni. Il Glossario del facility management - ripreso in seguito nelle note di un saggio dal titolo «Recupero architettonico: problematiche e questioni di metodo» sul n. 51 di Territorio - tenterà di districare questa confusione attraverso le normative in atto, partendo da un approccio gestionale e strategico: «Restauro: combinazione di tutte le azioni [...] che intervengono su un edificio tutelato, finalizzate a mantenere le informazioni contenute nell'edificio e nelle sue componenti, l'integrità materiale e ad assicurarne la conservazione e la protezione dei suoi valori culturali. [...] Recupero: combinazione di tutte le azioni [...] che intervengono su un edificio costruito, finalizzate a mantenere o aumentare le informazioni residue dello stesso edificio. [...] Riuso: combinazione di tutte le decisioni [...] finalizzate a modificare l'utilizzo di un edificio o di sue componenti ambientali o, qualora non utilizzato, a definirne l'utilizzo. Può attuarsi anche senza opere edilizie, oppure con interventi di manutenzione, riqualificazione o restauro. Riqualificazione: combinazione di tutte le azioni [...] condotte su un edificio o sue componenti tecnologiche, finalizzate a modificare le relative prestazioni per farle corrispondere a nuovi requisiti richiesti [...]».

Alle soglie del nuovo secolo si affermerà negli studi urbani un interesse per le pratiche d'uso, un'attenzione al contesto non solo fisico e storico, ma ambientale e sociale, fino all'inserimento di questi aspetti all'interno del progetto di trasformazione.

Ancora oggi, è urgente la necessità di un'azione più consapevole da parte degli strumenti urbanistici e delle normative edilizie, per consentire la messa in atto di un riutilizzo di suolo e una occupazione di territorio nella migliore delle forme possibili, che privilegi la sostenibilità degli interventi alla condizione di privatizzazione o meno dei soggetti proponenti o alla tipologia di attività proposta. Soprattutto, emerge la necessità di una graduale continuità dei processi in atto.

L'evoluzione finora raccontata pone in evidenza una nuova variabile, tanto necessaria quanto strategica, all'interno dei processi di trasformazione: la dimensione temporale.

Un tema centrale che assorbe diversi contenuti di questo numero di Territorio riguarda l'identità degli spazi dell'architettura 
industriale nel periodo più legato al concetto di memoria, quello del secolo appena concluso. Il saggio introduttivo spiega non solo la necessità di "partire dalle peculiarità di ogni singolo caso", ma anche di "considerare il progetto di recupero come un processo di stratificazione di valori materiali e significati»: ne è un esempio la sorprendente attenzione dell'idea insediativa di Giuseppe Samonà per il complesso industriale della Centrale Tifeo. I contributi di questa sezione mostrano casi di riattivazione della vita di luoghi e architetture, riconsiderazione di nuovi usi e pratiche adatti alle necessità contemporanee. Nel contributo introduttivo si parla di 'disgiunzione' tra preesistenza e fruizione contemporanea, come risultato del rapporto conflittuale tra forma e contenuto dell'architettura, e di sporadici risultati in cui non si è costretti a confermarne l'attività d'uso. Nell'analisi per il progetto di recupero dell'Italcementi di Monselice si fa strada una necessaria 'flessibilità' nei processi di conversione dell'ex-area industriale, interpretata non solo attraverso le tipologie di intervento, ma «in termini di tempo (accelerazioni, rallentamenti, interruzioni)». Il saggio «Osservare da est. Cina, memoria (industriale) e paesaggio» spiega la relatività temporale in termini di 'brevità' dei processi di trasformazione di luoghi sacrificati a uno "svettante» modello di progresso, e in termini di durata «dal punto di vista delle numerose e sempre troppo ampie porzioni demolite di architettura minore».

Alla dimensione temporale non è estranea la velocità con la quale i materiali dell'industria diventano obsoleti e rischiano una loro sostituzione sempre più rapida. Ci parlano del tempo perfino alcune annotazioni veloci all'interno dei saggi sulla crescita del valore documentario della fotografia, così immediata nel prendere nota, ma così eterna nei suoi risultati.

Anche nella discussione sui Piani di emergenza, secondo Tema di questo fascicolo, è trattato l'aspetto della 'priorizzazione', cioè della sequenza ottimale con cui mettere in fila le operazioni necessarie; si discute di «medio e lungo periodo». A fronte della domanda, che sorge spontanea: «qual è la misura, in termini di tempo, dell'‘emergenza'?», la risposta di questi saggi e dei casi studio presentati è quella di considerare i Piani di emergenza non più come piani operativi delle forze di soccorso ma, con una visione più ampia, come strumenti di pianificazione strategica di un sistema di strutture che governino la fase dopo l'evento disastroso.

Anche l'Anteprima di questo numero riflette sulla natura della modernità, attraverso ben costruiti confronti di materiale iconografico, su «come l'ortodossia moderna, concepita con spirito internazionale» sia stata diversamente interpretata. Si ripresenta ancora la domanda: quando il moderno diventa 'antico', tale da essere considerato oggetto di attenzione conservativa? La trasformazione di alcuni esempi del patrimonio industriale dismesso ha dovuto fare i conti con la variabile tempo e con la durata di una serie di procedure che hanno lasciato in fondo alle priorità la realizzazione di un nuovo spazio urbano. Alcune ricadute dello spazio urbano as built rivelano soprattutto l'incompiutezza e la fragilità dell'idea di spazio sottesa a queste visioni.

Come avviene nella natura, esempio sempre e inevitabilmente presente anche per noi contemporanei, ogni trasformazione richiede tempo. In relazione alla conprensione sempre più necessaria di questa variabile nei processi di trasformazione, si è fatto strada il concetto di resilienza, sempre più adottato negli interventi di chi si occupa di questi temi: un concetto appartenente in origine ai mondi della fisica e dell'ingegneria, che misurano la capacità di un materiale di assorbire l'energia di deformazione in seguito a un urto. Dopo aver fatto un largo giro attraverso discipline quali la psicologia (la capacità dell'essere umano di superare una crisi), l'ecologia (la capacità di un sistema di ritornare allo stato iniziale dopo una perturbazione) o l'informatica (la capacità di resistere all'usura nel garantire una prestazione), il concetto di resilienza è arrivato alle discipline dell'architettura e dell'urbanistica: non a caso compare in entrambi i contributi della sezione Temi, seppure condotti con un'attenzione a scale diverse.

La resilienza offre alla condizione di crisi la possibilità di preludere a un fenomeno di miglioramento e a un orizzonte di rinascita; per una comunità come la nostra, significa la capacità di riorganizzarsi nell'eventualità di un cambiamento. Una capacità che abbiamo sempre avuto, ma che non ci accorgevamo di possedere; che adesso si mostra possibile e soprattutto suggerisce alle varie discipline un atteggiamento innovativo.

Applicata questa opportunità alla scala del territorio, e «attraverso una visione ecosistemica della realtà, la resilienza arriva nel campo della pianificazione guardando ai sistemi territoriali come organismi in continua trasformazione, capaci di (ri) organizzarsi in conseguenza di eventi naturali, fisici, culturali e socioeconomici per raggiungere livelli accettabili di efficienza», come ci ricorda l'apertura del saggio «Approcci territoriali integrati per i piani di emergenza».

La resilienza è un concetto che ha la sua forza interna nella durata e che lavora con la trasformazione. Non è necessario ricordare le meravigliose visioni di Ovidio nei suoi libri delle Metamorfosi per comprendere quanta fragilità sia insita nell'azione di trasformazione. La fragilità è nel cambio di ruolo e di identità, e questa condizione di fragilità può essere rinforzata o indebolita dall'azione del tempo. La fragilità dei processi raccontati in questo numero di Territorio risiede nella delicata trasformazione di identità, e nella capacità di interpretare criticamente il passaggio tra linguaggio moderno ed esigenza contemporanea, lasciando da parte quelle che Pagano avrebbe chiamato «esitazioni edilizie». 